Notes

\title{
Electrochemical Performance of a Glassy Carbon Electrode Anodized in 1-Octanol
}

\author{
Hatsuo Maeda, Tong-Xing LI, Masayuki Hosoe, Munenori Itami, \\ Yuji YamauchI and Hidenobu OHMORI
}

\author{
Faculty of Pharmaceutical Sciences, Osaka University, \\ Yamadaoka, Suita, Osaka 565, Japan
}

Keywords Octanol-modified glassy carbon electrode, anodic oxidative treatment, cyclic voltammetry, electrochemical detection, alkylammonium ion sensor, calcium ion sensor

We have recently found that a potential sweep treatment of a glassy carbon electrode at between 0 $+2.0 \mathrm{~V} v s$. Ag wire in an alkanol containing $1 \mathrm{M} \mathrm{H}_{2} \mathrm{SO}_{4}$ can modify the electrode surface with the alkanol probably via an ether linkage." A glassy carbon electrode anodized in 1-octanol (C8-GCE) acquired a hydrophobic membrane on its surface similar to a selfassembled monolayer of 1-octanethiol formed on a gold electrode. However, the surface condition was suggested to be totally different from that of a glassy carbon electrode anodized in $\mathrm{H}_{2} \mathrm{O}$ (W-GCE) by essentially the same procedure. In order to elucidate the nature of the C8-GCE surface in more detail, the electrochemical performance of C8- and W-GCEs was compared further. In this paper we report on the effects of $\mathrm{CaCl}_{2}$, butylamine hydrochloride, and hexylamine hydrochloride upon the voltammetric response of $\mathrm{Fe}(\mathrm{CN})_{6}{ }^{3-}$ at $\mathrm{C8}$ and W-GCEs, which is expected to introduce a new concept in designing a modified electrode as an ionsensor.

A C8-GCE and a W-GCE were prepared as follows. A mechanically polished GCE $(3 \mathrm{~mm} \phi)$ was anodized in 1-octanol or $\mathrm{H}_{2} \mathrm{O}$ containing $\mathrm{H}_{2} \mathrm{SO}_{4}(0.1 \mathrm{M})$ by controlled potential electrolysis at $+2.0 \mathrm{~V}$ vs. Ag wire, instead of the potential sweep method ${ }^{1}$, in order to precisely control the amount of electricity consumed during the treatment. After $5 \mathrm{mC}$ electricity was consumed the electrode was successively washed with $\mathrm{MeOH}$ and deionized water, and then cathodically treated by sweeping the potential five times from $0-$ $-0.5 \mathrm{~V}$ vs. SCE in $0.1 \mathrm{M}$ aqueous $\mathrm{KCl}$.

Upon cyclic voltammetry (CV) of an aqueous solution of $\mathrm{K}_{3} \mathrm{Fe}(\mathrm{CN})_{6}(5 \mathrm{mM})$ and $\mathrm{KCl}(10 \mathrm{mM})$ at the thusobtained $\mathrm{C} 8 \mathrm{-GCE}$, the electrode process of $\mathrm{Fe}(\mathrm{CN})_{6}{ }^{3-}$ used as a marker was completely depressed, which was restored by the addition of dodecyltrimethylammonium bromide. Although a similar behavior was observed at a W-GCE, the surfactant induced no change in the depressed voltammogram. As previously reported ${ }^{1}$, the voltammetric behavior at a C8-GCE could be explained by the formation of an insulating hydrophobic membrane at the surface through its anodic oxidation in 1octanol, while the surface of a W-GCE is suggested to have been covered by anionic functional groups, such as carboxylate $^{2}$, which induces a depressed response of the anionic marker at the W-GCE due to an electrostatic repulsion.

Since $\mathrm{Ca}^{2+}$ is well known to restore the suppressed voltammetric response of an anionic marker at an electrode modified with a membrane containing anionic functional groups ${ }^{3-6}, \mathrm{Ca}^{2+}$ can be used as a probe for the existence of anionic sites on an modified electrode. Thus, for each aqueous solution containing $\mathrm{K}_{3} \mathrm{Fe}(\mathrm{CN})_{6}$ $(5 \mathrm{mM}), \mathrm{KCl}(10 \mathrm{mM})$, and $\mathrm{CaCl}_{2}$ of various concentrations $(0,0.5,1,4,7,10 \mathrm{mM}), \mathrm{CV}$ was performed at both the C8- and W-GCEs at a sweep rate of $100 \mathrm{mV} / \mathrm{s}$. The effects of $\mathrm{Ca}^{2+}$ upon the electrochemical response of $\mathrm{Fe}(\mathrm{CN})_{6}{ }^{3-}$ was evaluated based on the ratio of the cathodic current at $0 \mathrm{~V}$ vs. $\mathrm{SCE}$ in the presence of $\mathrm{CaCl}_{2}$ $(I)^{7}$ to that in the absence of the additive $\left(I_{0}\right){ }^{8}$ As shown in Fig. 1 , an increase in the current ratio $\left(I / I_{0}\right)$ with the concentration of $\mathrm{CaCl}_{2}$ was observed at the W-GCE, demonstrating that anionic functional groups actually exist on its surface, as is generally accepted. ${ }^{2}$ Unexpectedly, the additive exhibited a similar effect at the C8-GCE, which was more pronounced than that at the W-GCE. These results seem to indicate that a C8GCE possesses more anionic sites than does a W-GCE, contradicting to our conclusion that a C8-GCE has a hydrophobic membrane solely comprising an uncharged alkyl group.

In order to disclose the origin of the phenomena at a C8-GCE, it was examined how the coexistence of butylamine hydrochloride (1) and hexylamine hydrochloride (2) affects the response of $\mathrm{Fe}(\mathrm{CN})_{6}{ }^{3-}$ depressed at $\mathrm{C} 8$ - and W-GCEs. The reasons for choosing the ammonium ions were as follows: (1) 1 and 2 have no surfaceactivating ability like that of dodecyltrimethylammoni- 


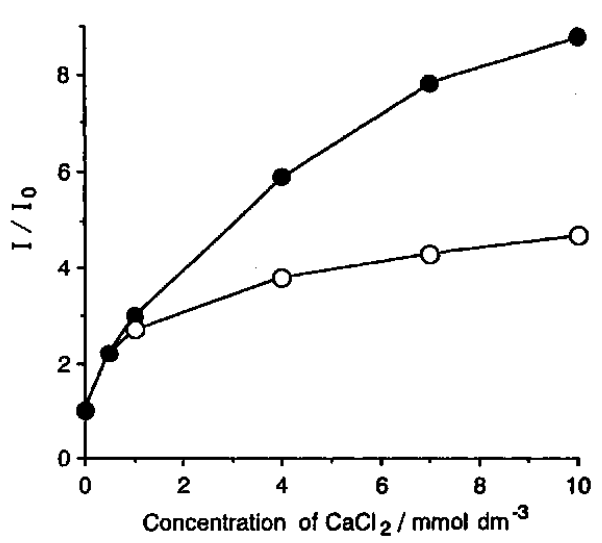

Fig. 1 Effects of $\mathrm{CaCl}_{2}$ on the response observed on the cyclic voltammetry of $\mathrm{K}_{3} \mathrm{Fe}(\mathrm{CN})_{6}(5 \mathrm{mM})$ in aqueous $\mathrm{KCl}(10 \mathrm{mM})$ at a C8-GCE (closed circles) and a W-GCE (open circles).

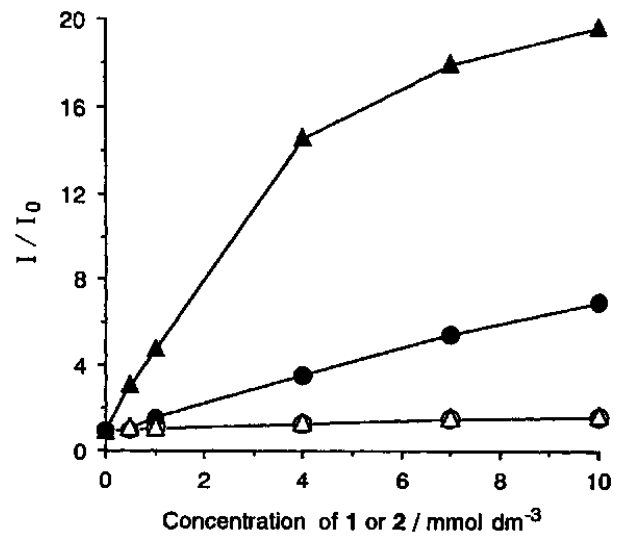

Fig. 2 Effects of butylamine hydrochloride (1) (circles) and hexylamine hydrochloride (2) (triangles) on the response observed on the cyclic voltammetry of $\mathrm{K}_{3} \mathrm{Fe}(\mathrm{CN})_{6}(5 \mathrm{mM})$ in aqueous $\mathrm{KCl}(10 \mathrm{mM})$ at a C8-GCE (closed marks) and a WGCE (open marks).

$\mathrm{Fe}(\mathrm{CN})_{6}{ }^{3-}$. This hypothesis is supported by the following findings: (1) the cations exhibited similar effects at a gold electrode covered with 1-octanethiol when the modification was performed by dipping a gold electrode in a dilute EtOH solution of the thiol for a short time, that is, when a less-packed membrane was deliberately formed on a gold electrode; (2) the influence of the cations on the voltammetric response of $\mathrm{Fe}(\mathrm{CN})_{6}{ }^{3-}$ was diminished at a C8-GCE prepared at the expense of more than $5 \mathrm{mC}$, that is, a GCE modified with a more-packed hydrophobic membrane. Although it was found to be very hard to reproduce a gold electrode covered with an appropriately less-packed membrane of 1-octanethiol, a newly polished glassy carbon electrode was furnished with almost the desired packing situation of the hydrophobic membrane in a highly reproducible manner by only controlling the amount of electricity during the anodic treatment in 1-octanol.

The present study implies that an electrochemical cation-sensing function will be conferred on a system consisting of an anionic marker and an electrode coated with a less-packed hydrophobic membrane. This expectation is very intriguing, since the indirect electrochemical detection of cations like alkaline earth ions has been achieved only by the response of an anionic marker at an electrode covered with a membrane containing anionic functional groups $s^{3-6,10}$, the preparation of which seems to often encounter some difficulty. Further studies to shed light on the mechanism for the observed phenomena and to examine the possibility of the present system as alkylamine- and alkaline earth ion-sensors are under way.

This work was supported in part by funds from Takeda Science Foundation. 


\section{References and Notes}

1. H. Maeda, Y. Yamauchi, M. Hosoe, T.-X. Li, E. Yamaguchi, M. Kasamatsu and H. Ohmori, Chem. Pharm. Bull., 42, 1870 (1994).

2. R. L. McCreery, "Electroanalytical Chemistry", ed. A. J. Bard, Vol. 17, p. 221, Marcel Dekker, Inc., New York, 1991.

3. M. Sugawara, K. Kojima, H. Sazawa and Y. Umezawa, Anal. Chem., 59, 2842 (1987).

4. M. Maeda, Y. Tsuzaki, K. Nakano and M. Takagi, $J$. Chem. Soc., Chem. Commun., 1990, 1529.

5. K. Takehara, Y. Ide and M. Aihara, Bioelectrochem. Bioenerg., 29, 103 (1992).

6. K. Takehara, Y. Ide, M. Aihara and E. Obuchi, Bioelectrochem. Bioenerg., 29, 113 (1992).

7. When the depressed response of $\mathrm{Fe}(\mathrm{CN}) 6^{3-}$ was recovered in the presence of the additive to give a well-defined peak at a potential more positive than $0 \mathrm{~V}$, its peak current was employed as $I$.

8. Background corrections were not made on the values of $I_{0}$ and $I$, since the background currents, observed on CV for a solution containing only $\mathrm{KCl}(10 \mathrm{mM})$ at $\mathrm{C} 8$ and $\mathrm{W}$ GCEs, were less than $10 \%$ of $I_{0}$ values at both anodized electrodes.

9. One of the referees suggested that the observed effects may originate from the cation exchange between $\mathrm{K}_{3} \mathrm{Fe}(\mathrm{CN})_{6}$ and $\mathrm{CaCl}_{2}, 1$, or 2, which allows $\mathrm{Fe}(\mathrm{CN})_{6}{ }^{3-}$ to permeate the hydrophobic membrane. We agree that the proposal is plausible. At present, however, it is not clear why such an ion-extraction takes place only at a lesspacked hydrophobic membrane.

10. N. Nakashima, T. Taguchi, Y. Takada, K. Fujio, M. Kunitake and O. Manabe, J. Chem. Soc., Chem. Commun., 1991, 232.

(Received August 26, 1994)

(Accepted October 28, 1994) 